\title{
First impressions revisited
}

$\mathrm{T}$ he Afghan Troop Medical Center is a quaint building with rosebushes lining the walkway and a well-groomed lawn. The first clue that this hospital is very different from any back home is that the lawn is tended by an old man, on his hands and knees, carefully cutting the grass handful by handful with a curved knife.

We are here to provide medical advice and mentoring to Afghan physicians, physician assistants, medics and preventive medicine officers; the 87 staff here serve the Kabul Military Training Center, a 10 000-person base east of Afghanistan's capital. As we enter the front door, the first thing that hits us is the overpowering smell of unwashed bodies. The narrow hallway is lined with crouching Afghan soldiers patiently waiting to be assessed. Soldiers enter the examination rooms in groups of three and four to be seen by an Afghan physician. This clinic sees 300 to 500 outpatients in the run of a day.

Further down the hall are two large treatment rooms, one for officers, the other for enlisted men, each with 23 beds. Any patient who needs observation will stay here for up to 48 hours and almost every patient receives intravenous fluids. Each bed has one or two soldiers lying on unwashed sheets.

These were our initial impressions, until the afternoon on July 4, 2012. Not far from the Troop Medical Center is the Haidari Propane and Gas Company. On that afternoon, their five 40000 -ton tanks exploded. Enormous mushroom clouds of fire and smoke billowed into the air. No one knew what was happening, or what was going to happen next. Soldiers and civilians ran, many with their clothes in flames, and in their effort to seek safety, attempted to scale barriers lined with razor wire.

The Troop Medical Center was overrun. The Afghan Chief of Medicine, Colonel Shir-Afzal was at home when the explosions started. He quickly got in his car to drive to the clinic, but in the confusion and chaos the roads became

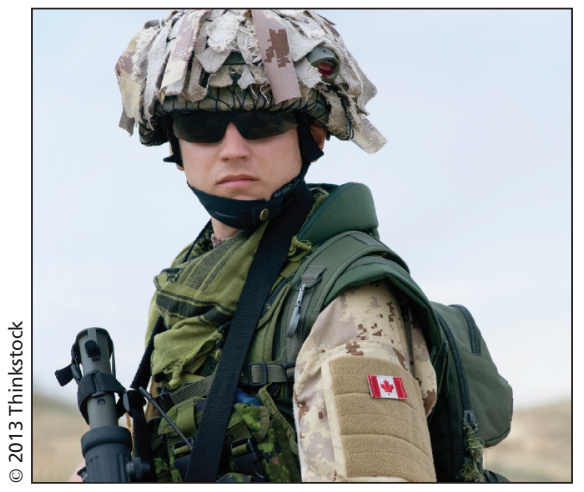

impassable. He abandoned his car and ran the rest of the way.

A Canadian and Australian medical team set out from inside our coalition camp to help. When we arrived, hundreds of patients were lying in lines on the front lawn. Shir-Afzal and his medics quickly triaged the most seriously wounded to be brought inside. This was no easy task. With so many patients with burns and lacerations, it would have been easy to overlook someone in need of life-saving care. Many of these Afghan medics had seen combat; however, none had dealt with this many casualties at once. ShirAfzal and his team methodically worked through the night, and by the time it was all over, they had treated 294 patients.

This mass casualty incident, as well as numerous less dramatic interactions with Colonel Shir-Afzal and his staff, offered us a different perspective of the Troop Medical Center. Our first impressions of a crowded, unclean and backward clinic were wrong.

The hallways are muddy, but that is to be expected when hundreds of soldiers, straight out of the training fields, pass through them every day and cleaning supplies are limited. Antibiotic prescriptions might seem too short, but this makes patients with continued symptoms return for reassessment and prescription refills, which ensures that as few pills as possible are wasted. The automatic administration of IV fluids is partially because most of these soldiers are dehydrated from their training, but more importantly this ensures that all the medics who rotate through this clinic are proficient at intravenous cannulation for times when they are faced with trauma. The sheets are dirty, because the nurses and clinicians have to wash them by hand.

We worked with our Afghan counterparts for nine months, and together made substantial progress. We helped them develop their vaccination program, acquire a washer and dryer for their linen, initiate daily team rounds, develop trauma bays and a continuing medical education program that included weekly grand rounds and electrocardiogram rounds. Had we treated these health care providers based on our first impressions, we never would have been as ambitious or accomplished all of this.

When we first came to this clinic, we judged it on the basis of what it lacked. What we missed is how much these doctors, nurses and medics care for their patients. Colonel Shir-Afzal and his staff make very little money and have to fight for every piece of equipment they need, but they are determined to give their patients the best care they can with what little they have.

\section{Captain Shane Smith MD MSc}

Family physician

Canadian Forces Health Services Centre Atlantic

Halifax, NS

Master Corporal Brad Studham

Preventive medicine technician

2 Field Ambulance

Petawawa, Ont.

The authors were deployed to Kabul, Afghanistan as part of the NATO training mission between February and November in 2012 .

\section{CMAJ 2013. DOI:10.1503/cmaj.122096}

Do you have an opinion about this article? Post your views at www.cmaj.ca. Potential Salon contributors are welcome to send a query to salon@cmaj.ca. 